\title{
Dynamic Characterization and Damping Control of a MEMS Structure
}

\author{
Ilgar Veryeri, Ipek Basdogan \\ Department of Mechanical Engineering, Koc University, Istanbul, Turkey 34450
}

\begin{abstract}
Micro Electro Mechanical Systems (MEMS) are among the new and emerging technologies of the future and have many applications in different disciplines. This study presents the dynamic characterization methods that we use to identify the modal parameters of a MEMS device and also the techniques that can be implemented to change the modal parameters. A micro scanner mirror was chosen as the case study to demonstrate the developed methodologies. Initially, the micro mirror was dynamically characterized using experimental modal analysis techniques to identify the modal parameters such as resonance frequencies and mode shapes. Then, it was introduced in a velocity feedback control loop to alter the effective damping of the structure. This method proves to be a very efficient method to alter the modal damping of a micro structure, especially when high quality factors are required for MEMS applications.
\end{abstract}

Keywords: MEMS design, quality factor, effective damping, dynamic characterization

\section{INTRODUCTION}

Micro Electro Mechanical Systems (MEMS) is among the new and crucial technologies of the future and has many applications in different disciplines. In this study, a velocity feedback control strategy is implemented to a MEMS device to alter the effective damping characteristics of the structure. A 1D torsional Flame Resistant (FR4) scanner was chosen as the case study to demonstrate the control strategy [1] (see Figure 1). Micro scanners are used for many applications ranging from laser-pointers, barcode readers to raster scanning laser displays. Fast scanning speeds and high scan angles achieved by the micro scanner technologies make them a good candidate for this type of applications. The mirror used in this study is actuated by Lorentz's forces created by an external magnetic field and an alternating current [2]. The mirror is used to deflect a modulated light beam on an image plane to create a display. The scanning angle, operation frequency and quality factor are among the design parameters of the micro scanners that should be highly taken into consideration.

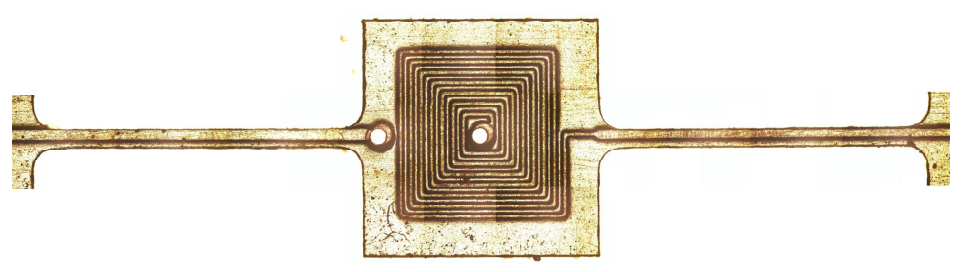

Fig. 1. Electromagnetic Actuated FR4 Scanner

If the torsional scanner is modeled as a spring-mass-damper system, its equation of motion can be written as

$$
\begin{aligned}
& I \ddot{\theta}+b \dot{\theta}+k \theta=T_{o} \cos \left(\omega_{R} t\right) \\
& \omega_{n}=\sqrt{\frac{k}{I}} \\
& Q=\frac{I \omega_{n}}{b}
\end{aligned}
$$


where $\theta$ is the mechanical rotation angle of the mirror, $I, b, k$ and $Q$ are the effective moment of inertia, damping coefficient, spring constant, and quality factor of the mirror for the $\mathrm{n}^{\text {th }}$ vibration mode, respectively. $\omega_{n}$ is the natural frequency at that mode; $\omega_{R}$ is the corresponding resonance frequency; and $T_{o}$ is the amplitude of the external driving torque.

The quality factor, $Q$, is an important design parameter as it describes the sharpness of the mirror response $[3,4,5]$. It is an inherent characteristic that influences the stability of the mirror. In this study, a velocity feedback control strategy is implemented to the FR4 Scanner to improve the quality factor of the first resonance frequency of the mirror. The main idea behind the standard damping control (Q-control) is to modify the quality factor (ie: effective damping) of the structure by adding a feedback signal to the actuation signal as given in Eq. 2 [6]. Then, the equation of motion of the system can be written as

$$
\begin{aligned}
& I \ddot{\theta}+b \dot{\theta}+k \theta=T_{o} \cos \left(\omega_{R} t\right)+G \dot{\theta}+H \theta \\
& I \ddot{\theta}+(b-G) \dot{\theta}+(k-H) \theta=T_{o} \cos \left(\omega_{R} t\right) \\
& k^{*}=(k-H) \\
& \omega_{n}^{*}=\sqrt{\frac{(k-H)}{m}} \\
& Q^{*}=\frac{I \omega_{n}^{*}}{(b-G)}
\end{aligned}
$$

where $\mathrm{G}$ and $\mathrm{H}$ determine the amount of velocity and position feedback gain, respectively. Equation 2 shows that pure velocity feedback $(\mathrm{H}=0)$ only modifies the $\mathrm{Q}$ factor of the system, whereas any position feedback leads to the variation of the $\mathrm{Q}$ factor as well as the natural frequency, $\omega_{n}$.

In order to achieve high performance requirements, modal characteristics of the mirrors must be well-understood. For that purpose, before implementing the velocity feedback control, we will dynamically characterize the micro scanner using experimental modal analysis techniques and identify the first natural frequency and the mode shape of the system. Then, we will introduce the mirror into a velocity feedback control loop and demonstrate that we could change the effective damping of the system by applying Q control. The applied control strategy appears as a direct-velocity feedback. Indeed, the additional controlling torque induced by the magnetic field is proportional to the transverse velocity and not to the rotational velocity of the corresponding structure. Since the scan angle of the micro mirror is really small, the rotational velocity can be approximated by the transverse velocity.

\section{DYNAMIC CHARACTERIZATION OF THE MICRO SCANNER}

The homebuilt experimental modal analysis setup in our laboratory includes a Polytec Laser Doppler Vibrometer (LDV), a laser positioning system, a microscope, a CCD camera, and a data acquisition system [7] (see Figure 2). The LDV controller provides signals and power for the sensor head, and processes the vibration signals with $0.15 \mu \mathrm{m} / \mathrm{s}$ resolution and it can detect signals up to $10 \mathrm{~m} / \mathrm{s}$. It has an upper frequency limit of $1.5 \mathrm{MHz}$. A CCD camera, a microscope scan unit and a X-Y stage are used for exact positioning of the laser beam for velocity measurements. 


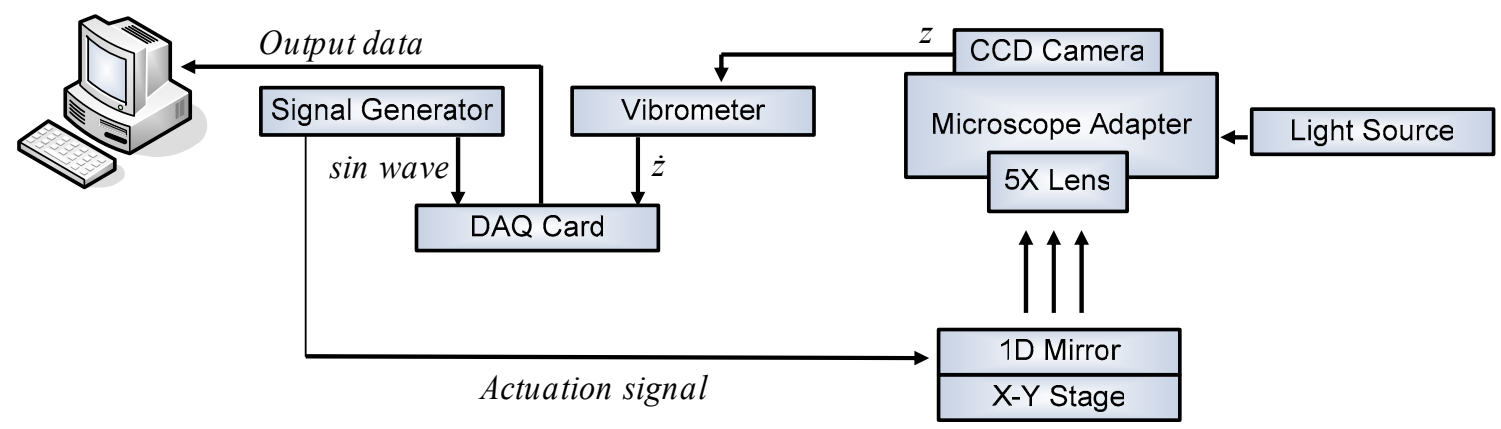

Fig. 2. Schematic of the experimental modal analysis setup (without velocity feedback)

At the first step of the experimental procedure, the picture of the micro device is captured by the CCD camera. The picture of the mirror is then divided into grids for determining the measurement points. The 'grid' procedure allows the accurate positioning of the laser on the micro device]. The micro mirror is excited at wide range of frequencies and the velocity of the vibrating device is acquired through the use of a data acquisition card and a software code written in LabView [8]. The frequency response functions (FRFs) that relate the excitation input to velocity output are measured at predefined grid locations of the micro structure and then modal analysis is performed utilizing the MEScope software [9]. Modal parameters are estimated from the experimentally measured FRFs via curve fitting. Curve fitting is a process of matching a parametric model of an FRF to a set of measurement data. The unknown parameters of the FRF model are modal frequency, modal damping \& mode shape for each mode that is identified in the bandwidth of the measurements.

Figure 3 shows the first mode shape of the micro scanner obtained experimentally. A typical FRF plot is also given in Figure 4. The quality factor $Q$ of a system can be also defined as the ratio of the natural frequency to the bandwidth at half power. The quality factor of the current mirror is calculated through a frequency scan of the $1^{\text {st }}$ mode shown in Figure 4, and was found to be $Q=33.4$.

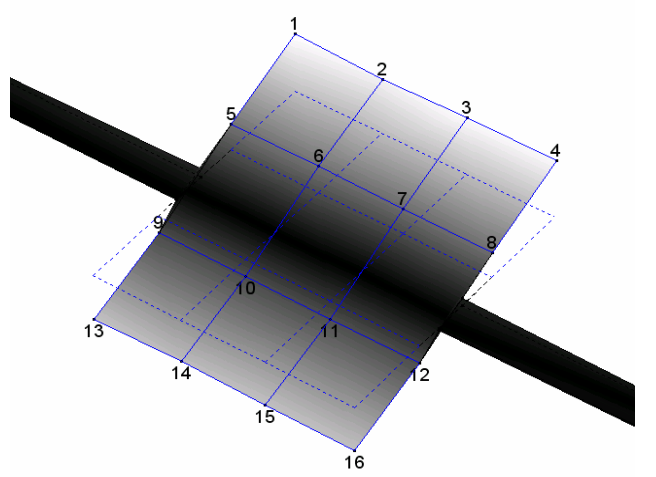

Fig. 3. $1^{\text {st }}$ mode shape $(\mathrm{Q}=33.4)$

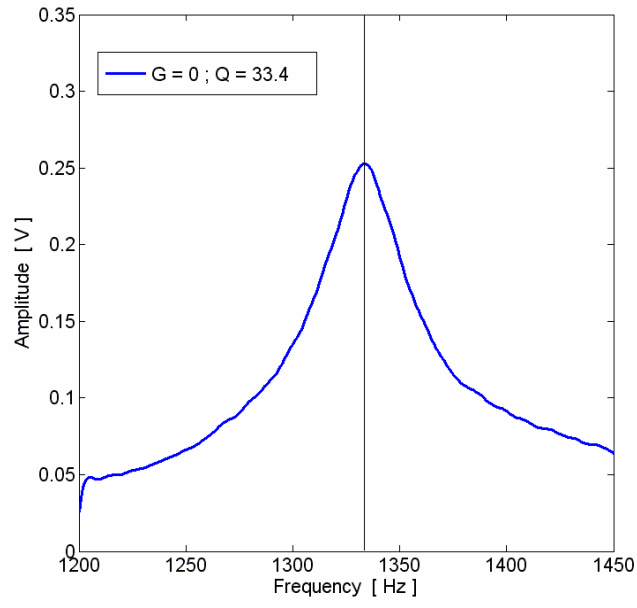

Fig. 4. Frequency sweep curve $\left(\omega_{\mathrm{R}}=1334 \mathrm{~Hz}.\right)$ 


\section{VELOCITY FEEDBACK CONTROLLER}

The mirror is introduced in a feedback loop as shown in Figure 5. The main components of the feedback circuit are the phase shifter and the gain amplifier, which are integrated into the vibration measurement setup described in the previous section. The analog phase shifter is integrated into the signal processing circuit to eliminate the intrinsic time delay in the LDV and hence to obtain a true velocity signal. Although the LDV used in our setup is equipped with a velocity decoder and directly measures the vibration velocity of the probe, a constant time delay in the measurement signal was observed, which causes an additional phase lag between the actuation and output signals. To eliminate the phase lag due to this constant time delay, the resistance in the phase shifter is adjusted in advance such that the output coming from the phase shifter is always in phase with the true velocity signal. The gain amplifier (AD633JN, Analog Devices, Norwood, MA) is used to alter the phase shifter output signal by a predefined gain, G. The gain is first adjusted by a $Q$ controller program, sent to the gain amplifier and then added to the actuation signal to modify the $Q$ factor of the mirror.

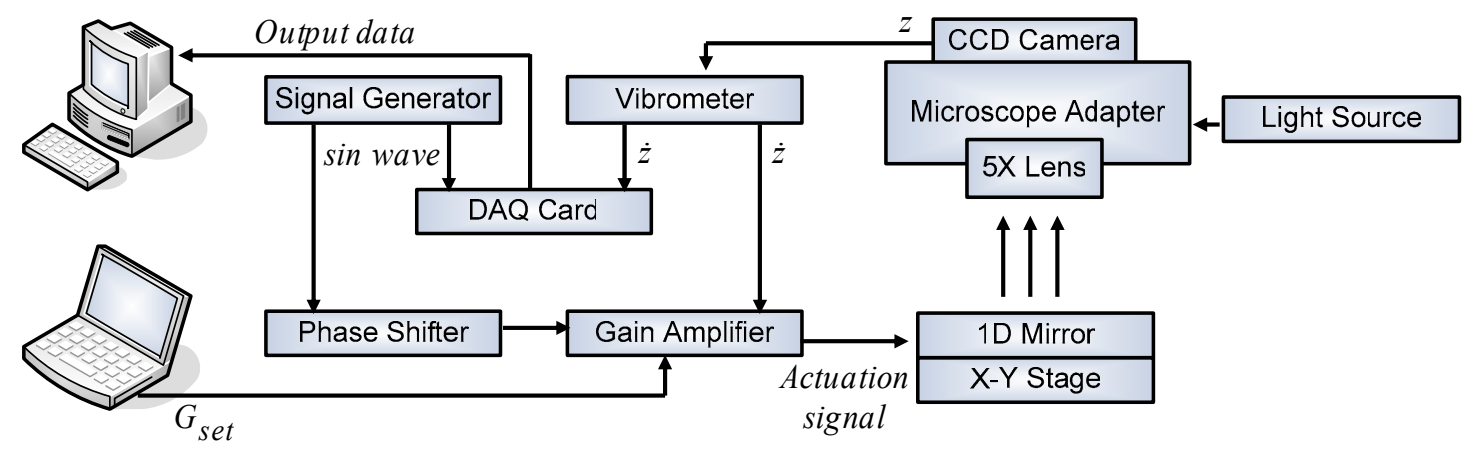

Fig. 5. Schematic of the experimental modal analysis setup (with the velocity feedback)

The first resonance mode of the mirror $\left(\omega_{\mathrm{R}}=1334 \mathrm{~Hz}\right)$ for different feedback gains is shown in the Figure 6 . It is observed that the effective $\mathrm{Q}$ factor of the mirror rapidly changes with the gain. The maximum gain $\mathrm{G}$ is just limited by the voltage range of the data acquisition board (National Instruments, PCI-6025E) used in the control loop (-10 to $+10 \mathrm{~V})$. The quality factor is increased from 33.4 to 111.2 , almost four times larger than the original system.

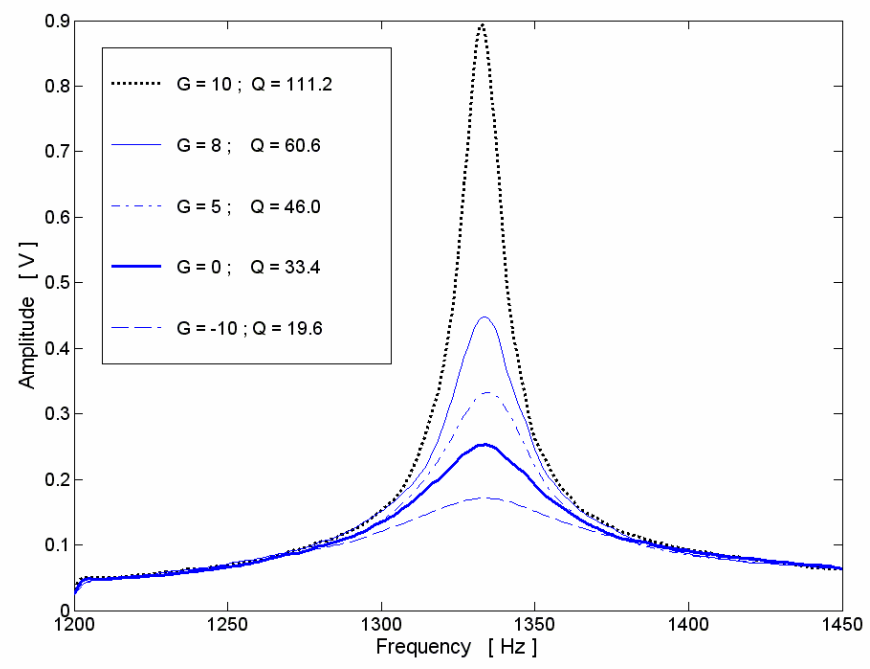

Fig. 6. Frequency sweep curves for different gain values. 


\section{DISCUSSION AND CONCLUSION}

This paper demonstrates the concept of changing the effective damping of a MEMS structure utilizing a velocity feedback control technique. The structure is first characterized dynamically and then the first mode of the structure is identified and visualized by applying modal analysis techniques to determine the mode shapes, modal frequencies, and damping coefficients using the transfer functions obtained at various points on the structure. Then, the Q-control is applied to the structure and the response is measured for various values of gain, $\mathrm{G}$.

We are currently working on the implementation of the displacement feedback control strategies on the MEMS structures in order to change the quality factor and also the resonance frequencies of the structure. Future study that may

follow the damping studies could be the investigation of the stability of the system when the gain factor is altered while the mirror is under operation.

\section{ACKNOWLEDGEMENTS}

This work is supported by European FP6 Program, Network of Excellence on Micro Optics and National Scientific and Technological Research Council of Turkey (TUBITAK).

\section{REFERENCES}

1. H. Urey, S. Holmstrom, A.D. Yalcinkaya, S. Isikman and C. Ataman, "FR-4 as a New MOEMS Platform", The 7th IEEE LEOS Conference, Hualien, Taiwan, 2007.

2. H. Urey, "High performance resonant MEMS scanners for display and imaging applications," Optomechatronic Micro/Nano Components, Devices, and Systems Conference, Proc.SPIE, Vol.5064, Philadelphia, Pennsylvania, October 2004.

3. M. Collet, V. Walter, and P. Delobelle, "Active damping of a micro-cantilever piezo-composite beam," Journal of Sound and vibration, Vol. 260, pp. 453-476, 2003.

4. Tomas R. Rodriguez, Ricardo Garcia, "Theory of Q control in atomic force microscopy", Applied Physics Letters, Vol. 82, pp.4821-4823.

5. Q-Control Information Booklet, NanoAnalytics, 2003

6. Ihsan Gunev, Aydin Varol, Sertac Karaman, Cagatay Basdogan, "Adaptive Q Control for Tapping Mode NanoScaling using Piezoactuated Bimorph Probe”, Review of Scientific Instruments 78, 043707, 2007.

7. O. Anac, I. Basdogan, "Model Validation Methodology for Design of Micro Systems", 8th Biennial ASME Conference on Engineering Systems Design and Analysis, ESDA 2006, July 2006, Torino, Italy.

8. http://www.ni.com/labview/, accessed in November 2006.

9. Me'scope VES operating manual, Version 3.0, September 2001 